\title{
Metamaterials for Microwave Radomes and the Concept of a Metaradome: Review of the Literature
}

\author{
E. Öziş, ${ }^{1}$ A. V. Osipov, ${ }^{1}$ and T. F. Eibert ${ }^{2}$ \\ ${ }^{1}$ German Aerospace Center (DLR), Microwaves and Radar Institute, Oberpfaffenhofen, Germany \\ ${ }^{2}$ Chair of High-Frequency Engineering, Department of Electrical and Computer Engineering, Technical University of Munich, \\ Munich, Germany
}

Correspondence should be addressed to E. Öziş; ezgi.oezis@dlr.de

Received 20 January 2017; Revised 23 March 2017; Accepted 9 April 2017; Published 20 July 2017

Academic Editor: Scott Rudolph

Copyright (C) 2017 E. Öziş et al. This is an open access article distributed under the Creative Commons Attribution License, which permits unrestricted use, distribution, and reproduction in any medium, provided the original work is properly cited.

\begin{abstract}
A radome is an integral part of almost every antenna system, protecting antennas and antenna electronics from hostile exterior conditions (humidity, heat, cold, etc.) and nearby personnel from rotating mechanical parts of antennas and streamlining antennas to reduce aerodynamic drag and to conceal antennas from public view. Metamaterials are artificial materials with a great potential for antenna design, and many studies explore applications of metamaterials to antennas but just a few to the design of radomes. This paper discusses the possibilities that metamaterials open up in the design of microwave radomes and introduces the concept of metaradomes. The use of metamaterials can improve or correct characteristics (gain, directivity, and bandwidth) of the enclosed antenna and add new features, like band-pass frequency behavior, polarization transformations, the ability to be switched on/off, and so forth. Examples of applications of metamaterials in the design of microwave radomes available in the literature as well as potential applications, advantages, drawbacks, and still open problems are described.
\end{abstract}

\section{Introduction}

Electromagnetic metamaterials are artificial compositions of resonant particles (metallic or dielectric) in a dielectric substrate, which are designed to control interaction of electromagnetic waves with the medium in a chosen wavelength region. The particles or inclusions are typically organized in a periodic array with the size of a unit-cell much smaller than the wavelength, so the incident wave interacts with the metamaterial as with an effectively homogeneous medium. Quasi two-dimensional metamaterial structures, involving just one layer of the unit-cells, are referred to as metasheets or metasurfaces. Metasheets are closely related to frequency selective surfaces (FSS), the difference being in the size of the unit-cells, which is comparable to the wavelength in FSS [1].

By properly choosing the shape and the size of the inclusions, the size of the unit-cells, and the substrate material, one can create metamaterials with effective electromagnetic parameters (permittivity, permeability, wave and surface impedance, etc.) that can be adjusted to almost any values, including those not encountered in nature. Metamaterials have the advantage of being tunable and tailorable, with a wide range of realizable electromagnetic properties. Such materials can be used to build antenna radomes with improved transparency and even with new features such as the property of being tunable or switchable, thus resulting in a new class of devices: metamaterial radomes or "metaradomes" $[2,3]$.

"Metamaterial covers" [4] for small antennas, aiming at improvement of transmission and directivity of the covered antennas, can be seen as metaradomes as well. These covers can be polarization-dependent to change the polarization state of the antenna $[5,6]$. Radomes involving FSS structures [1] are also linked to the concept of metaradome. Our focus in this paper is however on electrically large radomes in conjunction with metasheets $[7,8]$.

Metamaterials find applications not only in RF and microwave engineering but also in acoustics, nanotechnology, photonics, optics, and medical engineering. The literature about the design and applications of metamaterials is 


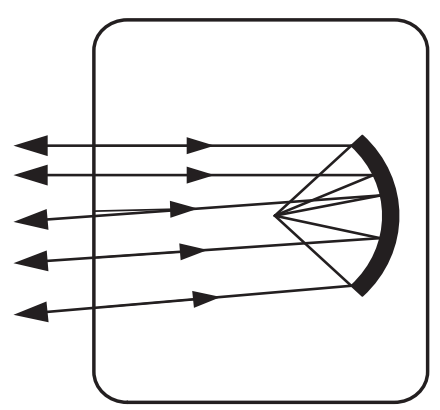

(a)

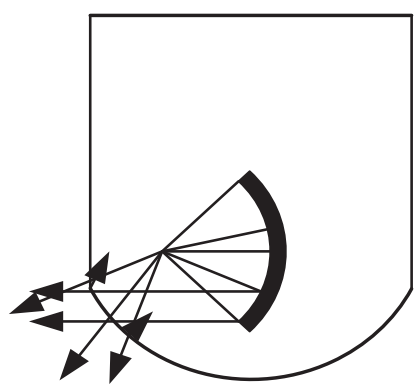

(b)

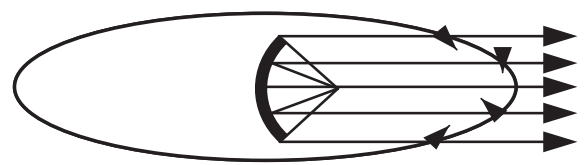

(c)

FIGURE 1: Different radome shapes: (a) cylindrical; (b) cylindrical-hemispherical; (c) spheroidal or ogival [16].

enormous, and we do not even attempt to review it. The reader is referred to the books [9-15]. The goal of this paper is rather to present a short overview of the basic features of metamaterials which can be relevant to applications in microwave radomes, give a collection of relevant references, and introduce the concept of the metaradome, a microwave radome with improved and extended functionality, made entirely from a metamaterial or involving a thin metamaterial sheet (metasheet).

The paper is organized as follows. Conventional microwave radomes are addressed in Section 2, basic features of electromagnetic metamaterials in Section 3, FSS in Section 4, metasheets in Section 5, tunable metamaterials in Section 6, and known examples of applications of metamaterials to microwave radomes in Section 7. The concept of the metaradome, advantages, and drawbacks of the use of metamaterials in radome applications are addressed in Section 8.

\section{Microwave Radomes}

The essential role of a radome is to form a protective cover between the antenna and the surroundings with minimal impact on the electrical performance of the antenna. An ideal radome should be fully transparent and lossless, that is, electrically invisible. Radomes should satisfy electrical, structural, and mechanical requirements. From the electromagnetic point of view, reflection, diffraction (at discontinuities in the radome, e.g., ribs), and absorption (in the radome material) should be accounted for when designing a radome [16-18].

The choice of the shape and material of the radome is typically determined by the application. Radomes are often curved structures made from ceramics and composites with high values of permittivity, which may result in a degraded performance of the antenna $[19,20]$. Several differently shaped curved radomes are shown in Figure 1. In aerospace applications, the radome design must be compliant with aerodynamic requirements as well. The shape has a direct impact on the electrical characteristics of every radome.

The major parameters that characterize the radome performance are transmittance and boresight error. Transmittance is the ratio of the energy flows transported by the waves to and from the radome wall. It determines, for example, the operation range of a radar. Transmittance is a function

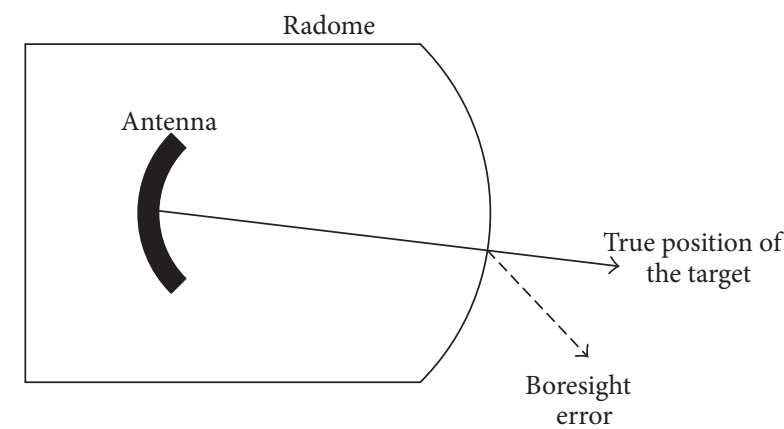

FIGURE 2: Boresight error in a radome: angular displacement from correct position [22].

of frequency, wall construction (thickness and material constants), incident angle, and polarization. The boresight error depends on more parameters: antenna position, the shape of the antenna aperture and the radome nose, frequency, polarization, and wall construction (sometimes with an integrated lens for phase compensation) [21]. The boresight error is caused by refraction of electromagnetic waves at the nonparallel interior and exterior sides of the radome wall with the result that a target is seen at an angularly changed, wrong position with respect to the antenna (Figure 2). This effect deforms the antenna beam [22].

Thus, radomes can negatively affect the antenna performance by distorting the amplitude and phase in the main beam, bringing more sidelobes, boresight errors, and a decreased bandwidth [17].

\section{Metamaterials}

There are various definitions of metamaterials in literature such as the following:

(i) "Materials whose permeability and permittivity derive from their structure" $[23,24]$.

(ii) "Structures composed of macroscopically scattering elements" [24, 25].

(iii) "A new class of ordered nanocomposites that exhibit exceptional properties not readily observed in nature. These properties arise from qualitatively new 
response functions that are not observed in the constituent materials and result from the inclusion of artificially fabricated, extrinsic, low dimensional inhomogeneities" [24, 26].

(iv) "Macroscopic composites having a man-made, threedimensional, periodic cellular architecture designed to produce an optimized combination, not available in nature, of two or more responses to a specific excitation. Each cell contains metaparticles. The metamaterial architecture is selected to strategically recombine local quasistatic responses or to combine or isolate specific nonlocal responses" [24, 27].

(v) "Artificial periodic structures with lattice constants much smaller than the wavelength of the incident radiation" [28].

So, a metamaterial is usually a periodic array of resonators (inclusions), placed in a substrate, with the size of the unitcells much smaller than the wavelength of the incident radiation. On the scale of the wavelength, the structure appears to be almost homogeneous with effective values of material constants: permittivity $\varepsilon$ and permeability $\mu$. By adjusting the parameters of the periodic structure (shape and size of the inclusions and period of the array) the effective material constants can be tailored to desired values, including those not encountered in natural materials. The use of resonant particles as the inclusions results in a frequency-dependent response (dispersion) of every metamaterial.

Most of natural materials have positive permittivity and permeability, and therefore they are referred to as "doublepositive" (DPS) media. Since the wave vector $\mathbf{k}$ and the power flow vector $\mathbf{S}$, given by the cross product of the electric field $\mathbf{E}$ with the complex conjugate magnetic field $\mathbf{H}(\mathbf{S}=(1 / 2) \mathbf{E} \times$ $\mathbf{H}^{*}$ ), are always codirected, such media are also referred to as "right-handed" media [14, 15].

Some materials have either a negative permittivity or a negative permeability. This results in a purely imaginary value of the wavenumber $k=\omega \sqrt{\varepsilon \mu}$, which implies strong attenuation of waves and makes such media unsuitable for the transmission of waves. When both $\varepsilon$ and $\mu$ are negative, the wavenumber $k$ is negative but real valued and propagation of waves is again possible. The vectors $\mathbf{k}$ and $\mathbf{E} \times \mathbf{H}^{*}$ are oppositely directed in this case, but $\mathbf{E}, \mathbf{H}$, and $\mathbf{S}$ still form a right-handed system. Such media, called "double-negative" (DNG) or "left-handed" media, do not exist in nature, but they can be created artificially $[10,14]$. The diagram in Figure 3 illustrates this classification.

A great variety of resonators can be used as inclusions in designing metamaterials. Some of them, shown in Figures 4 and 5, are split-ring resonators (SRR), stepped impedance resonators (SIR), ring resonators, open complementary splitring resonators (OCSRR), broadside coupled split-ring resonators (BC-SRR), nonbianisotropic split-ring resonators (NB-SRR), double slit split resonators (DSSRR), omega particles, and chiral particles [29-33]. Omega and chiral particles (Figure 5) couple electric and magnetic fields, which may result in bianisotropic metamaterials.

Depending on the choice of inclusions, one can create isotropic, anisotropic, chiral, and bianisotropic materials. The

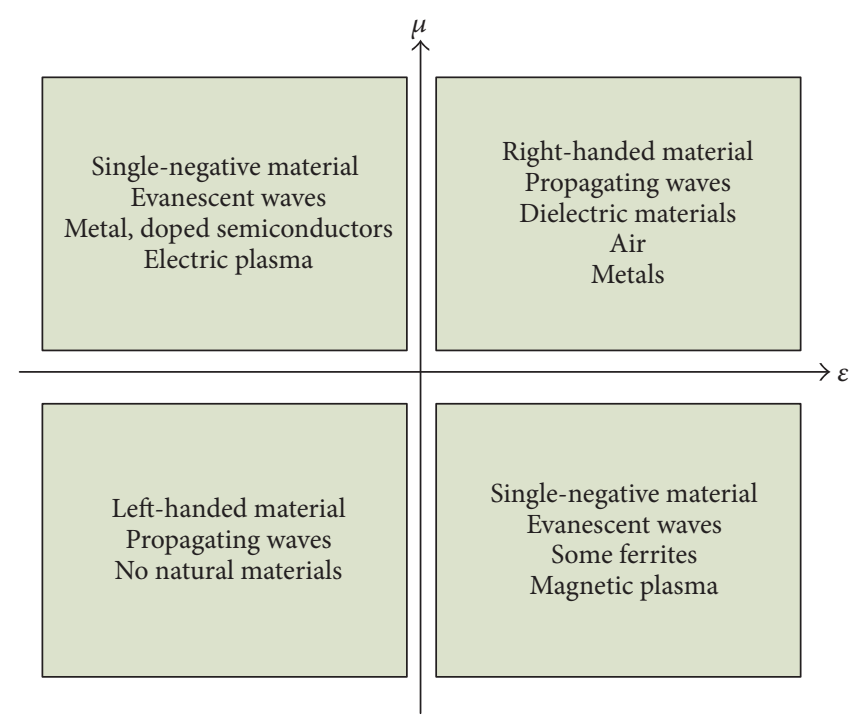

FIGURE 3: Classification of media according to permittivity and permeability $[15,94,95]$.

effective permittivity and permeability may approach any desired values, for example, be negative or close to zero $[13,34,35]$.

\section{Frequency Selective Surfaces}

Radomes require thin layers rather than bulk materials. Efficient design concepts that rely on the surface effects rather than on volumetric effects have been first developed and realized for radio waves as frequency selective surfaces (FSS) [36].

FSS are built from many small patterned metal elements, which are of resonant size, that is, comparable to or slightly smaller than the wavelength [1]. Typical geometries of the elements are dipoles, Jerusalem crosses, and square and circular loops [37]. These elements are printed on a dielectric substrate and are similar to printed circuit boards (PCB). For changing the frequency characteristics, FSS can be mounted on a biased ferrite substrate or PIN diodes can be connected to the elements so that the structure can be made tunable or switchable [37]. Such FSS devices as transmitarrays [38] and reflectarrays [36] have become widely recognized tools in modern radio-antenna engineering. A consequence of the resonant size of the elements is however the presence of side lobes in the reflected and transmitted fields, which is a characteristic feature of FSS.

\section{Metasurfaces and Metasheets}

Quasi two-dimensional metamaterials that consist of thin layers of inclusions with the thickness much smaller than the wavelength are called metasurfaces, metasheets, or metafilms, depending on whether the layer is penetrable or not. An example of a penetrable structure is shown in Figure 6 . In contrast to FSS, the size of resonators and unit-cells in metasurfaces and metasheets is much smaller than the wavelength, which eliminates the grating lobes in the reflected and transmitted fields. 


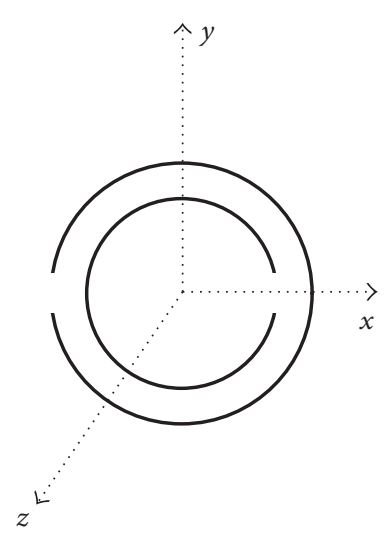

(a)

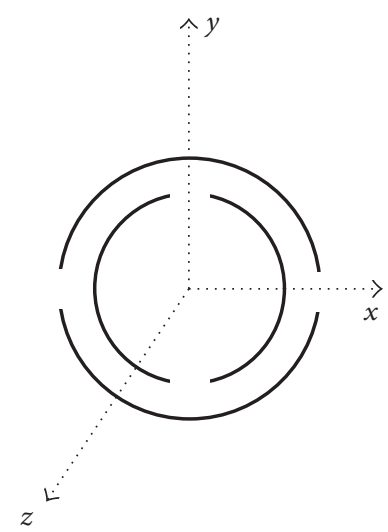

(b)

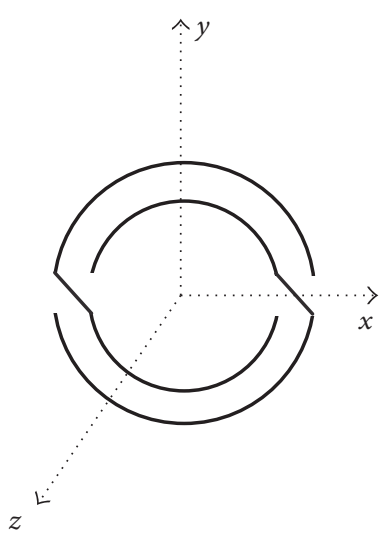

(c)

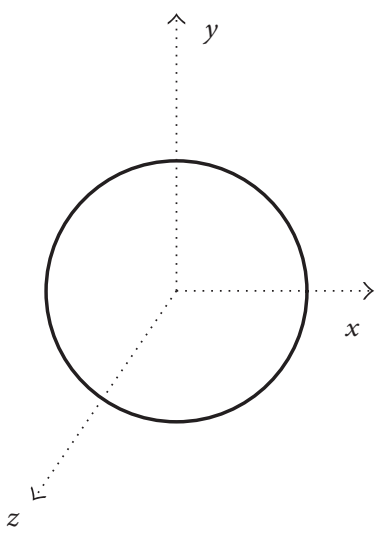

(d)

FIGURE 4: Examples of resonators used in the design of metamaterials: (a) conventional SRR; (b) double slit split-ring resonator (DS-SRR); (c) nonbianisotropic split-ring resonator (NB-SRR) [29]; (d) solid ring.

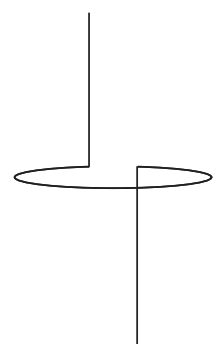

(a)

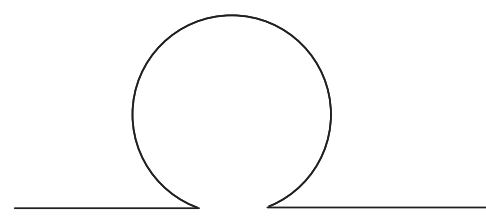

(b)

Figure 5: (a) Chiral particle; (b) omega particle [96].

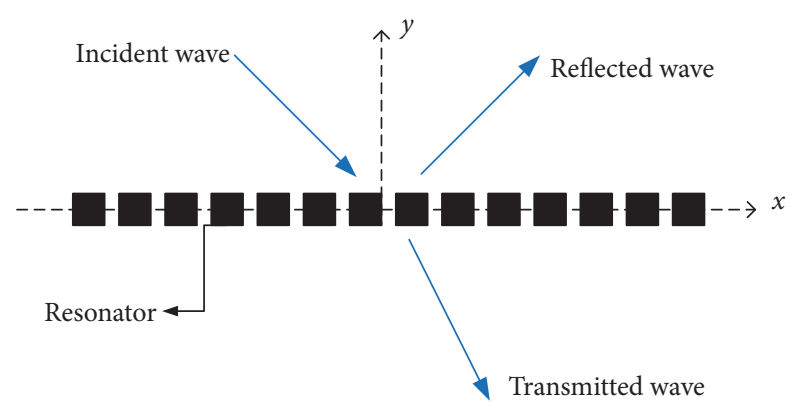

Figure 6: A metasheet (metafilm) consisting of arbitrarily shaped resonators periodically arranged in the $x-z$ plane [97].

At microwave frequencies, impenetrable metasurfaces are realized as a dense periodic texture of small elements printed on a grounded slab. A penetrable metasurface (called metasheet) involves a periodic planar distribution of small elements placed on a very thin host medium penetrable for electromagnetic waves [39-42].

Despite their small thickness, metasheets can fully control reflection, absorption, and transmission of electromagnetic waves (e.g., plane, surface or guided), including polarization, phase, and amplitude of the transmitted wave [35, 43-45]. As shown in literature, it is theoretically possible to design devices for almost arbitrary manipulations of plane waves, which results in such devices as self-oscillating teleportation metasheets, transmitarrays, double current sheets, and metasheets formed by only lossless components [46].

The interaction between an incident field and the field scattered at an electrically small particle is described by electric and magnetic polarizabilities, which can be dyadics or tensors in the most general case. When particles are arranged in an array, the field illuminating a given particle consists of the incident wave and the field scattered at surrounding particles. This effect can be accounted for by effective polarizabilities that relate the incident field to the dipole moments induced on the particles in the array. Transmission and reflection coefficients are expressed in terms of the effective polarizabilities, and one can search for optimal arrays of optimal particles by imposing desirable requirements on the transmission and reflection coefficients, for example, by minimizing reflection and absorption and maximizing transmission of a given wave. To get more degrees of freedom, bianisotropic particles are needed, which is the most general type of particles, polarizable by both electric and magnetic fields and with magnetoelectric coupling [31, 47]. Chiral particles are a special case of bianisotropic particles, and bianisotropy is a combination of anisotropy with magnetoelectric coupling [48].

In the design of metasheets capable of influencing in a desired way the propagation of an incident wave, the Huygens principle can be applied [49]. According to the Huygens principle, each point on a wave front may behave 

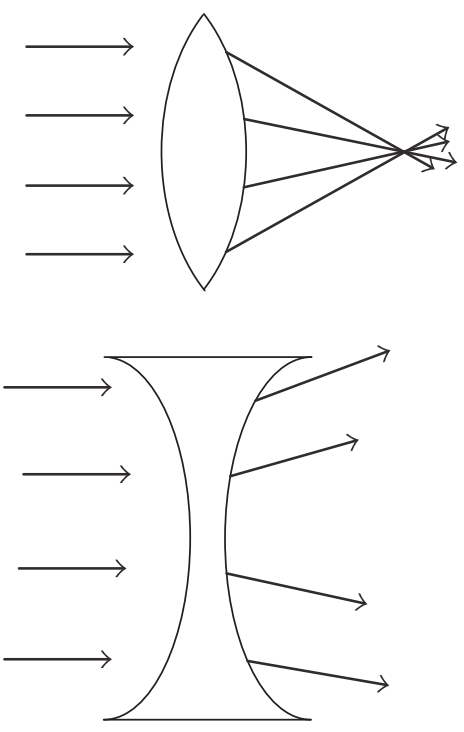

(a)
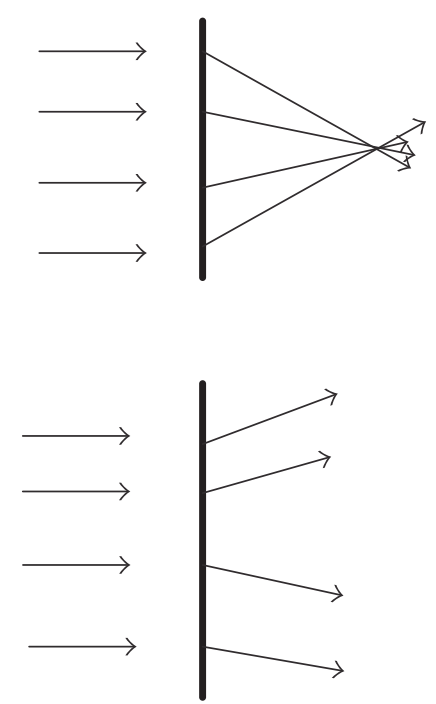

(b)

Figure 7: (a) Conventional optical lenses should be curved for focusing or defocusing the light; (b) planar Huygens' metasheets behave like a lens by locally controlling electric and magnetic currents induced on the surface $[49,51]$.

as a secondary source of spherical waves, and the sum of all secondary waves regulates the travel and the form of the wave $[49,50]$.

Conventional optical lenses can be replaced with planar metasheets as shown in Figure 7 [51]. Several metasheets combined together may work as a metamaterial with a negative index of refraction. Such structures can operate as a superlens providing images with the details finer than those allowed by the diffraction limit as shown in Figure 8 [52]. Realization of a superlens is however difficult as small deviations from ideal values of material parameters may significantly distort the image, for example, [53].

Transparent metasheets can be useful for the design of radomes since every radome must ensure high transmission and low reflection and absorption within the operation frequency band of the enclosed antennas. A suitable radome wall can be created by using a bulk metamaterial or by adding a metasheet to the existing radome from a conventional material. Metamaterial particles are embedded in a host dielectric medium, and their shape and size are adjusted so as to achieve desired values of the radome parameters $[7,8]$.

\section{Tunable Metamaterials}

Reconfiguring the structural components of a metamaterial changes the effective (macroscopic) properties of the metamaterial. By doing so, it is possible to tailor metamaterials for a required purpose. This property is a remarkable attribute of metamaterials [34]. If the effective properties can be changed in real time, then the metamaterial is referred to as tunable [54].

Tunability can be achieved in a number of ways, including (i) electrical tuning of permittivity, (ii) electrical tuning of permeability, (iii) magnetic tuning of permittivity, and (iv) magnetic tuning of permeability, as well as simultaneous tuning of both permittivity and permeability $[55,56]$. These changes can be achieved by adjusting the geometry of the unit-cell, for example, size, orientation, and position of resonators, or the material of the substrate. All these changes can be applied at the same time [54].

Another approach is the use of active lumped elements, such as non-Foster active elements [57], micro-electromechanical systems (MEMS) [58, 59], pin diodes [60, 61], varactors [62], or voltage-controlled capacitors [63]. For example, a voltage-controlled capacitance can affect the resonant frequency, so a varactor can be utilized for real-time tuning. A pin diode affects resistance. Using a non-Foster loading can influence the width of the frequency band. Liquid crystals can be inserted in unit-cells of metamaterials, and the refractive index of the substrate is tuned by applying a bias electric field which influences the orientation of liquid crystal molecules $[64,65]$. FSS structures can also be combined with liquid crystals, and frequency shift can be realized with a bias voltage [66].

Still another approach is the use of small resonators with ferroelectric insertions (e.g., barium-strontium-titanate, lead-strontium-titanate, silver-tantalate-niobate, and barium-stannate-titanate) exploiting the temperature dependence of the permittivity of the material $[67,68]$.

Converting the radome wall into a tunable structure can bring great flexibility in controlling the properties of the covered antenna. For example, low-loss metasurfaces with reconfigurable reflection and partially reflective surfaces studied in $[59,69]$ can be used for this purpose.

\section{Examples of Metaradomes}

This section gives examples of metamaterial devices and components that can be used in the design of microwave radomes. The aim of every metaradome is to improve the electromagnetic response of the enclosed antenna and eliminate the negative effects of conventional microwave radomes (Section 2). Table 1 summarizes the characteristic features of the designs. 


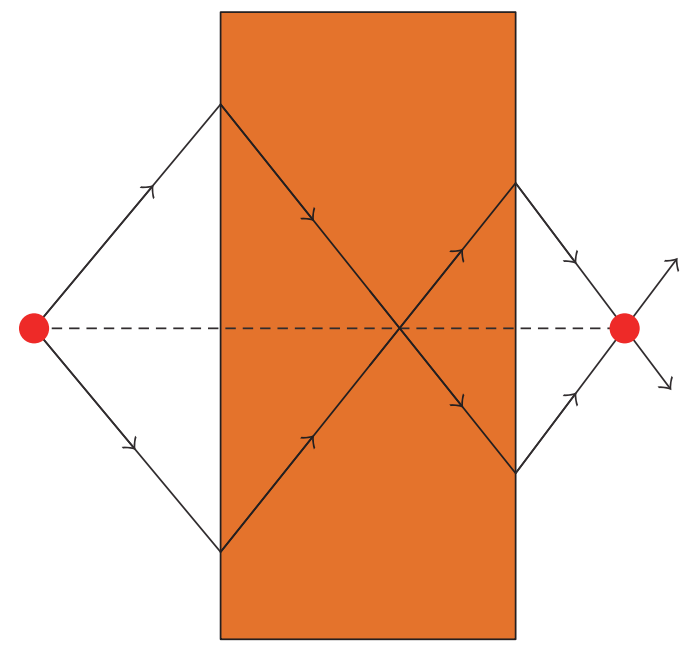

Figure 8: The operation of a superlens. Propagating and evanescent fields from the source go through a planar slab of negative-index material to form an image of the source. The image includes details finer than the wavelength, which cannot be realized by positive-index lenses [52].

TABLE 1: The list of the characteristic features of the metaradome designs described in Section 7.

Design Characteristic feature

(1)
PV cells as a metamaterial layer, dual band (lower band: $70 \mathrm{MHz}$ bandwidth around $1185 \mathrm{MHz}$, upper band: $3400-3600 \mathrm{MHz}$ ), high gain (17.3 and $6.6 \mathrm{dBi}$ at 3.5 and $1.23 \mathrm{GHz}$, resp.) [70]

Omega and reversed-omega elements in the unit cell, improved transmission between 13 and $17 \mathrm{GHz}$ [72]

DPS and DNG media, gain increase to $6 \mathrm{dBi}$, beamwidth reduction by $37.5 \%$ [73]

Highly transparent at normal incidence, reduced reflection at oblique incidence, no phase change upon transmission [74]

Gain enhancement through 9 subwavelength holes by about $3.4 \mathrm{~dB}$ [75]

A 4-dB improvement in radiation pattern for blind spot angle, wide-angle impedance matching, blind spot mitigation [3]

DPS and DNG layers, gain increase by $3.45 \mathrm{~dB}$, increase in the directivity by $2.9 \mathrm{~dB}$, bandwidth improvement [78]

Refractive index smaller than unity, CP antenna, 3-dB improvement of the gain, increase of the bandwidth [79] Heat-resistant structure, stable transmission at different incident angles, bandwidth increase from $10 \mathrm{GHz}$ to $12 \mathrm{GHz}$ by changing the size of unit cells [80]

Uniaxial medium with large permittivity along the anisotropy axis, operation on the near field, TM polarization transparent, broader radiation pattern, nearly eliminated interference within the cavity [81]

Polarization- and frequency-selective metasheets in $\mathrm{X}$ and Ka bands [82]

Disc-shaped electrically large metasheet, a hybrid approach (PO/FEM) to calculation of transmission [8]

Control of the phase of the transmitted wave by DC voltage, scanning over a wider range of radiation angle (up to $60^{\circ}$ steering angle), $8 \mathrm{dBi}$ maximum gain, beamwidth less than $7^{\circ}$ [83]

Nonreciprocal ferrite structure, improved isolation, 21-dB difference between S12 and S21 at $8 \mathrm{GHz}$, transmission along one direction and attenuation along the opposite direction [85]
7.1. Passive Metaradome Designs. In the first 12 examples, passive metamaterials are used in the design of the radomes. They are passive in the sense that active lumped elements, which are described in Section 6, are not utilized. In the examples below, mainly the type of unit-cells and the number of layers are modified. The choice of the particles plays an important role in shaping the electromagnetic response of the structures. The particles are combinations of electrically small metal wires and rings which interact with the incident wave as electric and magnetic resonant dipoles, respectively. Introducing gaps in the ring part of the particle (split-ring resonators and omega particles) results in a particle which combines the inductive and capacitive behavior, and this is a way of influencing the resonant frequency of the structure. The effective permittivity and permeability of the substrate are changed according to the shape of the particles. Thus, 
by adjusting the geometry and size of the particles the resonance frequency can be controlled and the passband can be expanded.

The following examples are taken from the current, available studies of the use of metamaterials in the design of microwave radomes. In each example, a different electromagnetic parameter such as gain, transmission, resonance frequency, band width, and multiband feature is improved by tailoring the geometry, size, material, and position of the particles. Because of the ease of numerical simulations, planar radomes are mostly studied in the literature.

(1) A panel of photovoltaic (PV) cells can be used as a metamaterial layer with a dual operation band [70]. The PV panel is used as a subwavelength selective periodic structure. In the first band, it behaves like a transparent sheet. In the second band, it behaves as a semitransparent sheet to ensure a high antenna gain. The structure is inspired by the concept of metamaterial Fabry-Pérot resonator [71].

(2) Differently positioned omega particles such as combinations of two or four omega and reversed-omega elements in the unit-cell can be used to design planar metamaterial radomes with an advanced performance. The omega-shaped particle is a bianisotropic particle. In this design, for eliminating this intrinsic bianisotropy, reverse-omega particles are combined with omega particles. The design described in [72] is characterized by good transmission between 13 and $17 \mathrm{GHz}$.

(3) Another design involving S-shaped particles is suggested in [73] to improve the antenna gain at a frequency around $5 \mathrm{GHz}$ (Figure 9). Multilayered models, which include two materials with negative and positive refraction indices, are used.

(4) Combining a conventional dielectric material, which is always right-handed, with a left-handed metamaterial can increase the transmittance. The metamaterial helps to compensate the phase change as a wave propagates within the structure, implying reflection and transmission without the phase changes [74].

(5) For modifying the antenna pattern and improving the antenna gain, a subwavelength hole in the middle of a Jerusalem-cross structure as shown in Figure 10 can be used. The structure, called "a gain enhanced antenna radome," concentrates electromagnetic fields and can change the antenna performance. The structure can play an important role in wireless communication [75].

(6) A metaradome can be designed to improve the response of a phased-array antenna at a blind condition. The blind direction of antenna arrays is the result of interaction between the Bloch array eigenmodes and the propagating surface waves or leaky waves [76]. The blind spot causes a failure of sending and receiving signals in that space angle region [76]. The scan blindness is also discussed in [77].
A metaradome has been designed as a wide-angle impedance matching slab in [3] to alleviate this problem.

(7) In [78], a radome with an operation frequency around $2 \mathrm{GHz}$ is designed with different layers of positive refractive index materials and an SRR-based negative permeability metamaterial. By adjusting the electrical and geometrical parameters, the gain, the bandwidth, and the directivity can be improved.

(8) Many communication systems operate in the circular polarization mode. The advantage of circular polarization (CP) is that it eliminates the need to continuously align the two slits of the apertures; otherwise, the power of the system has to be increased. Cross$\mathrm{S}$-shaped inclusions can be used for CP antennas. For example, a design described in [79] involves the crossS-shaped inclusions printed on an FR4 substrate with the size of the unit-cell $18 \mathrm{~mm} \times 18 \mathrm{~mm}$ to create a metamaterial structure with an effective index of refraction, which is positive but smaller than unity and close to zero between $3.3 \mathrm{GHz}$ and $3.7 \mathrm{GHz}$. According to the Snell law, rays leave such a slab almost normally to its surface. As a result, antenna gain and bandwidth have been improved.

(9) A structure suitable for high temperatures is described in [80]. It consists of an Ag (silver) microstructure pressed between two layers of quartz glass. The transmission can be controlled by changing the geometry of the Ag grid and the size of the unit-cells.

(10) A layer of a uniaxial medium with very large permittivity along the anisotropy axis can be used to transmit both propagating and evanescent spatial harmonics of the source onto the outer interface of an antenna to eliminate the effects of diffraction. Such a medium can be realized as a block of metal wire media embedded in a dielectric material and used as a radome [81].

(11) A simple prototype of a polarization- and frequencyselective metaradome is described in [82]. The structure consists of a periodic array of copper strips printed on top of a $2 \mathrm{~mm}$ thick layer of the FR4 epoxy (Figure 11). The structure blocks the transmission of fields polarized along the strips in a particular frequency range. By changing the dimension of the strips and the size of the unit-cells the operation frequency range can be changed. Two designs have been manufactured and measured, one for the $\mathrm{X}$ band (Figure 12) and another for the Ka band (Figure 13). For the Xband structure, the length of wire is $8.21 \mathrm{~mm}$, the width of strip is $2 \mathrm{~mm}$, and the unit-cell size is $14 \mathrm{~mm}$. For Ka band, the dimensions of strips and unit-cells are smaller by the factor 3.48. The slight disagreement between the simulation and measurement is due to the fact that simulations assume an infinite planar periodic structure, whereas the manufactured plates are finite. 


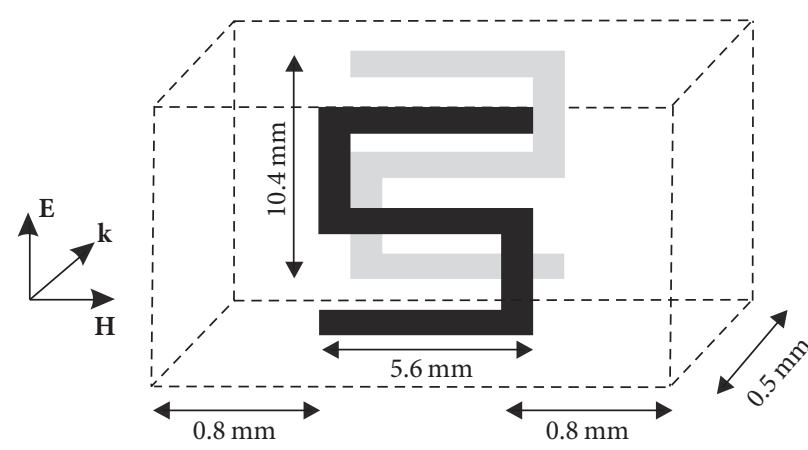

(a)

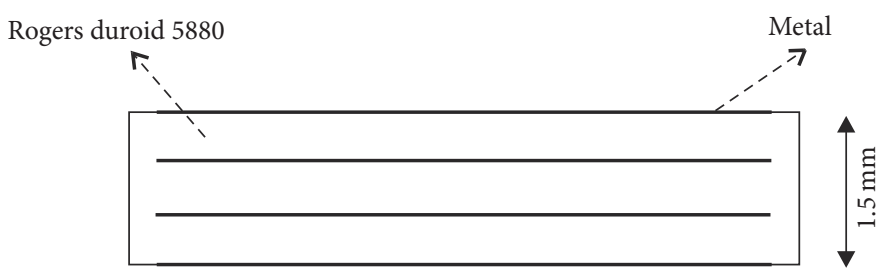

(b)

FIGURE 9: (a) Unit-cell structure; (b) structure for simulation [73].

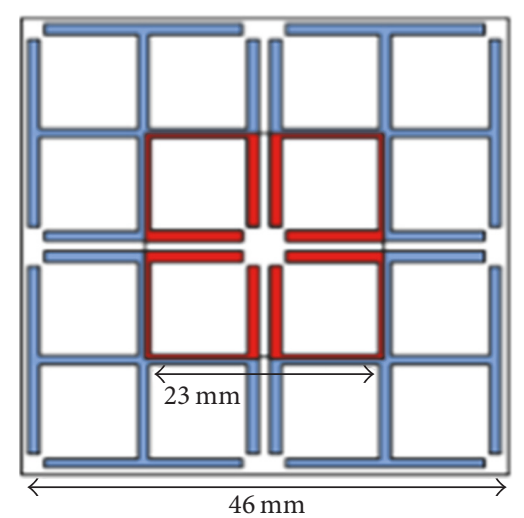

(a)

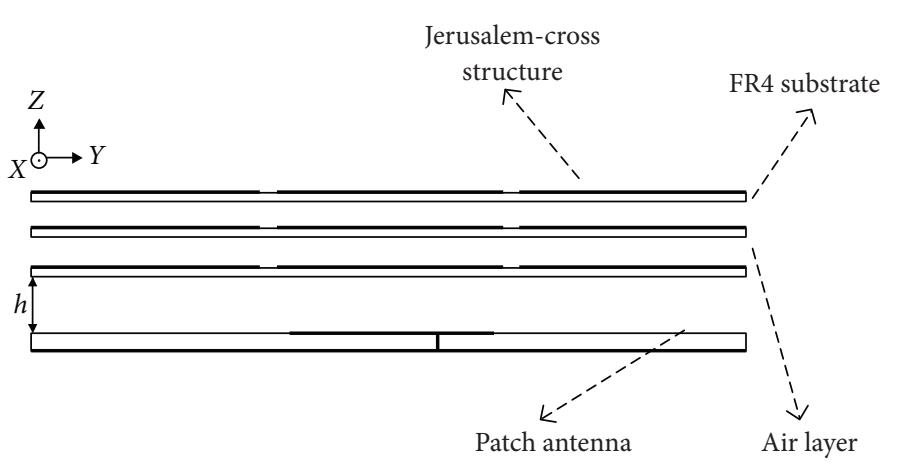

(b)

FIGURE 10: (a) Jerusalem-cross structure; (b) configuration of metamaterial radome and antenna [75].

(12) Calculation of transmission through metasheets of finite size is difficult because the assumption of periodicity of the structure is not applicable. Full-wave numerical simulations of such structures are limited to structures of several wavelengths in size. A hybrid approach combining Physical Optics (PO) approximation with transmission coefficients obtained from full-wave calculation for an infinite planar periodic structure is proposed in [8]. The unit-cells should be sufficiently small to justify the use of homogenized transmission coefficients. To estimate the accuracy of the hybrid approach, a circular metasheet consisting of copper rings on the FR4 substrate (Figure 14) was used as a test configuration. The diameter of the disc $(160 \mathrm{~mm})$ and the wavelength $(30 \mathrm{~mm})$ are such that the full-wave simulation is still possible and the structure is sufficiently large in order the PO method to apply. There is a good match of full-wave simulation and PO calculation (Figure 15).

7.2. Metaradome Designs Involving Active/Lumped Elements. In the next two examples, active metamaterials are used in the design of the radome surface. Active metamaterials mean that active lumped elements or ferrite insertions (Section 6) are included in the designs to control the electromagnetic response. An active element, varactor, is used in example (13). The ferrite is used in example (14). The use of ferrite insertions may lead to a nonreciprocal radome. Both examples show metaradomes that can be externally controlled.

(13) A metasheet with tunable refractive index can be realized by using varactors in the resonant cells of the metamaterial (Figure 16). The varactor with applied DC voltage behaves as an adjustable capacitor, which can be used to control the effective refractive index by changing the resonance frequency. Such a radome can control the phase of the transmitted wave by applying different DC voltages to different zones of the metamaterial slab since the phase shift depends on the bias voltage [83].

(14) Transmission can be permitted in one direction and suppressed in the opposite direction by a nonreciprocal metasheet. This feature comes from Faraday rotation, when electromagnetic waves propagate parallel to the applied bias field or in the direction of remanence magnetization in self-biased magnetic materials. Ferrites have low eddy current losses, which make them suitable for the use in some electronic applications such as power generation, conditioning, and conversion. Ferrites are particularly useful for 


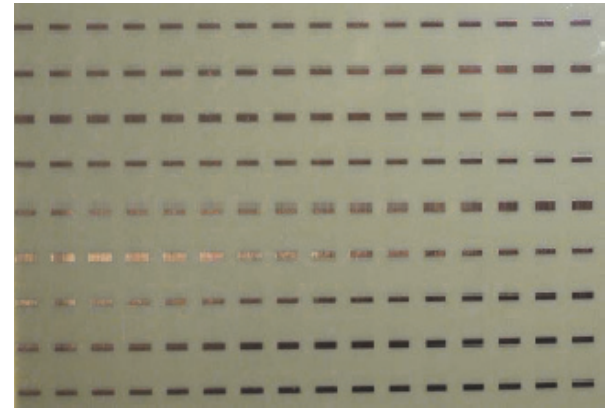

(a)

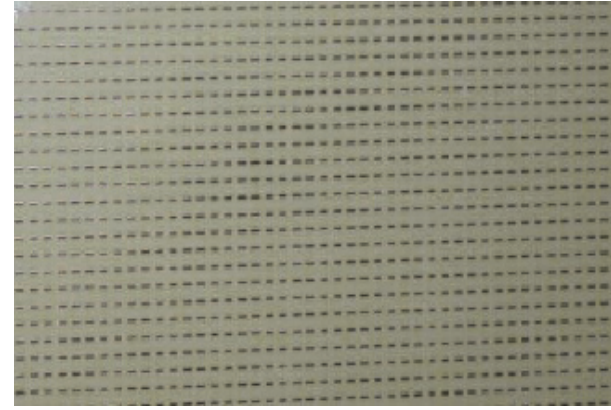

(b)

FIgURE 11: The X-band (a) and Ka-band (b) structures consisting of differently sized copper straight wires (strips) printed on top of the FR4 substrate.

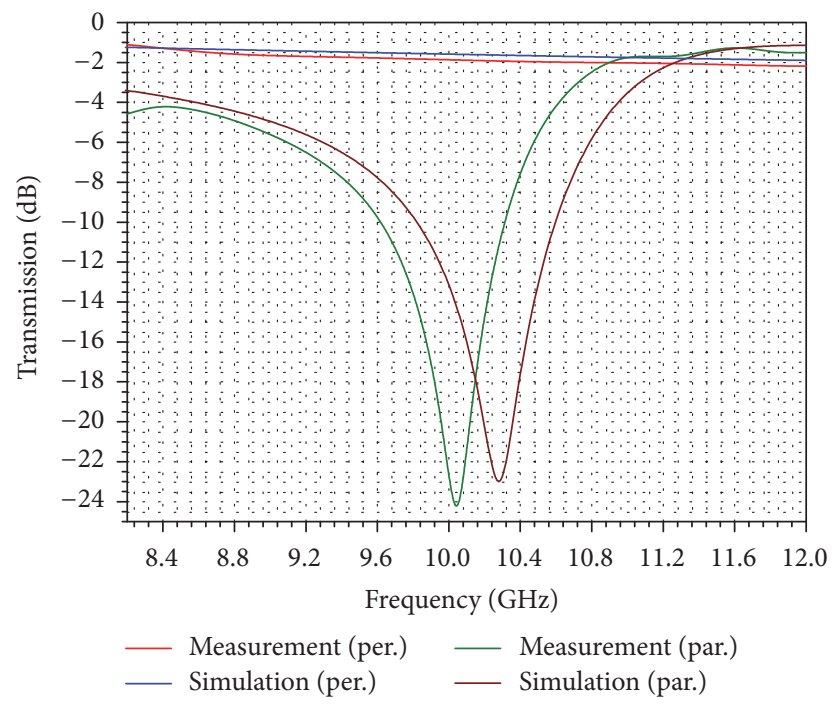

FIGURE 12: Measured and simulated (HFSS) transmission through the X-band structure.

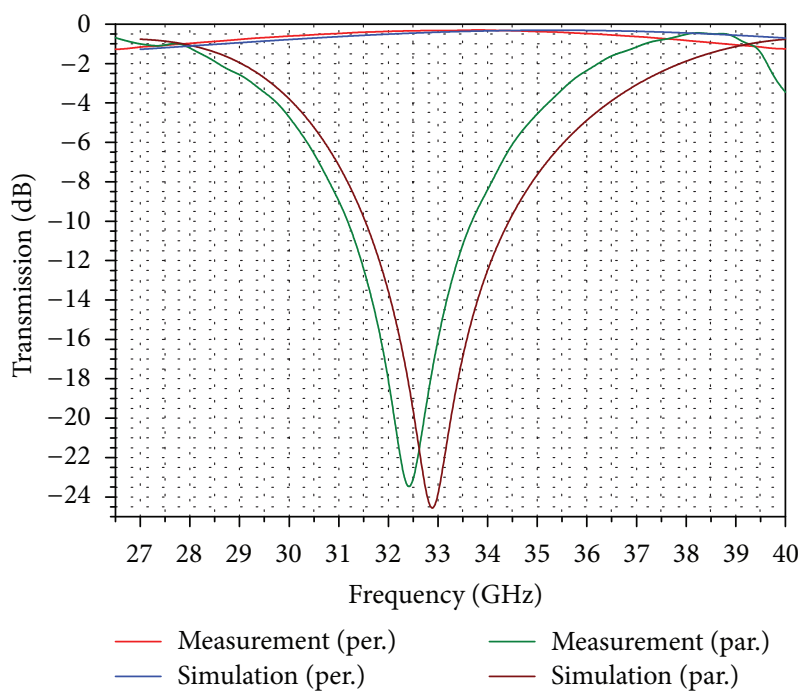

FIGURE 13: Measured and simulated (HFSS) transmission through the Ka-band structure.

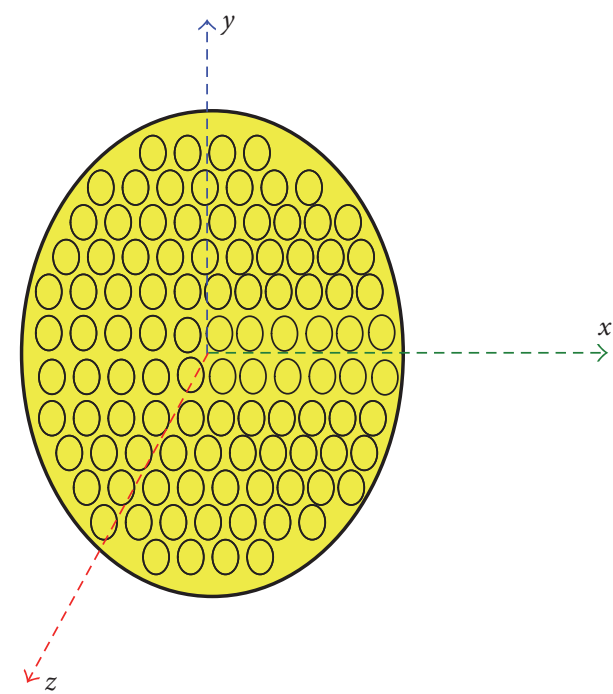

FIGURE 14: The circular electrically large metasheet in the $x-y$ plane. Copper rings are placed on the FR4 substrate [8].

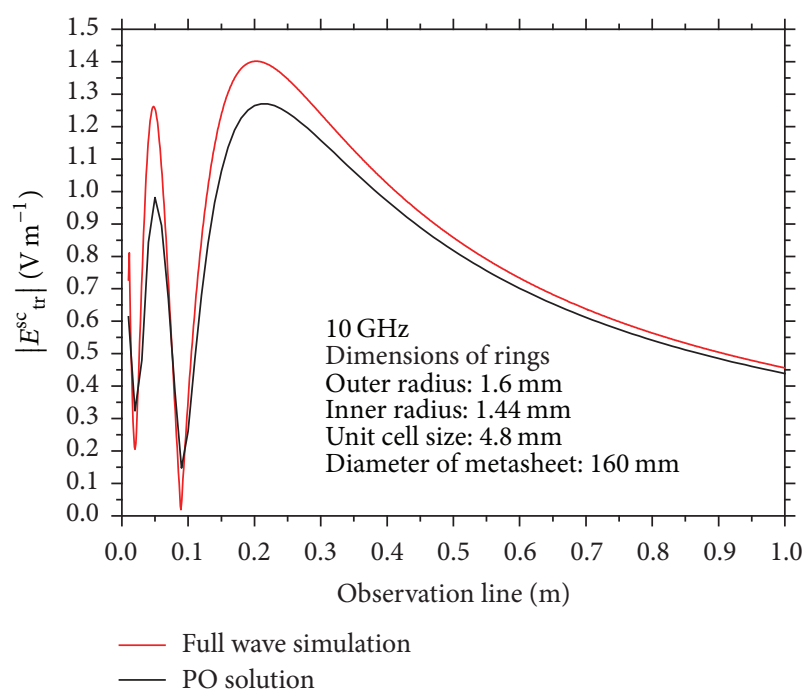

FIGURE 15: Scattered electric field along the observation line (along $z$-axis) in the shadow region behind the metasheet [8]. 


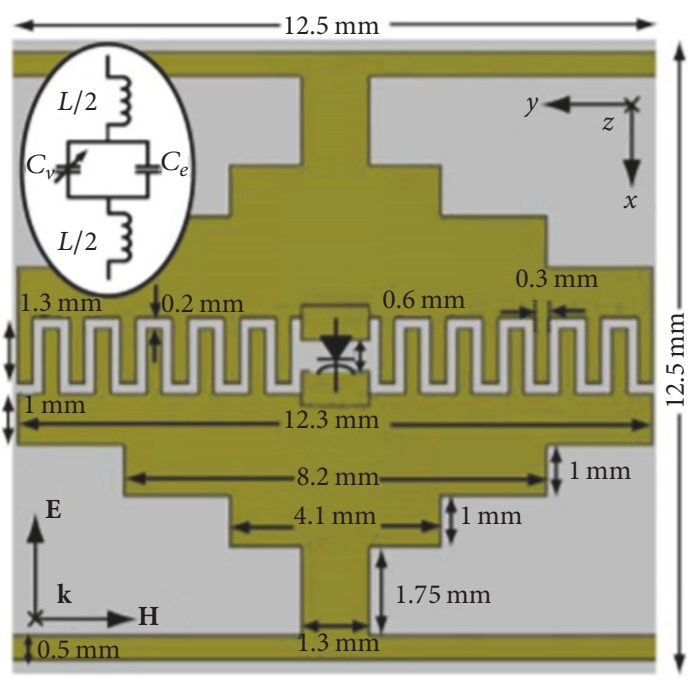

(a)

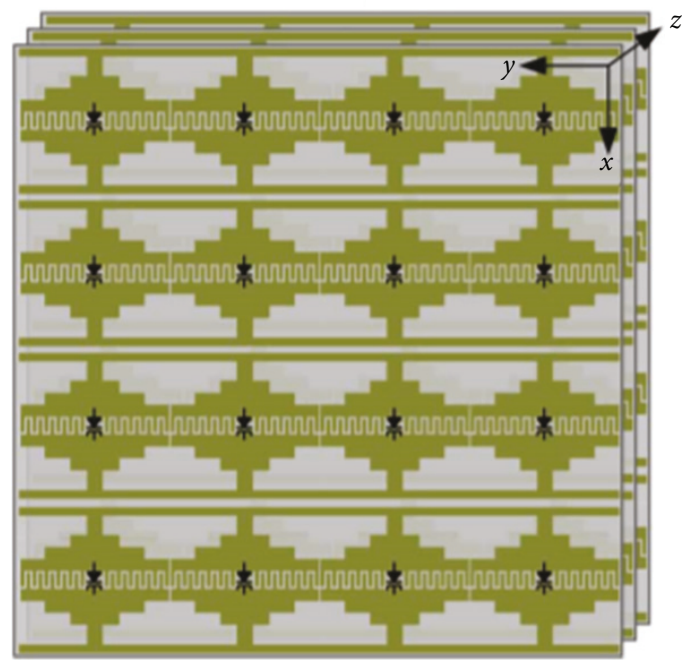

(b)

FIGURE 16: (a) Unit-cell of the realized metamaterial and its equivalent circuit; (b) metamaterial structure as a combination of unit-cells. The operating frequency is $4.7 \mathrm{GHz}[83]$.

microwave devices that need strong coupling to electromagnetic signals and nonreciprocal behavior [84]. In the design described in [85], highly conductive strip gratings are adjusted on each side of a ferrite layer. The strips behave as microwave polarizer. The direction of the polarization rotation depends on the direction of the applied bias field.

\section{Metaradomes: Pros and Cons, Potential Applications}

Metamaterials and particularly their quasi two-dimensional versions, metasheets, promise many important applications in conjunction with microwave radomes. A metasheet with tailored transmission, absorption, and reflection properties can be mounted on the wall of a radome to bring additional features and benefits such as correction of phase distortions, reduction of transmission losses, shaping the frequency dependence of the transmission, and making the radome tunable, including the ability of being switched on/off. Implementation of the concept may result in multiband radomes with reconfigurable frequency pass bands, on/off modes, enhanced out-of-band rejection and eventually in an enhanced antenna gain.

The problems to be solved are as follows:

(i) Most of the available realizations of metaradomes are planar, partly because of the relative ease of simulation (as infinite periodic structures) and partly due to the ease of manufacturing. Simulation of bounded and/or curved metamaterial structures is a difficult calculation problem because of the need to sample extremely fine subwavelength inclusions distributed over an electrically large area without the periodicity assumption. Simulation of curved metasurfaces is an open problem and a future research field. A possible approach is addressed in [8]. (ii) The limited bandwidth, which is due to the resonance behavior of the individual inclusions, is an inherent feature of metamaterials. A number of recent studies are devoted to the extension of the bandwidth of metamaterial absorbers but little is known about extending the bandwidth of transparent structures. It should be noted however that a narrow bandwidth is not necessarily a negative situation because narrowband systems are as necessary as wide-band systems in communication networks. For example, narrowband systems help to have the cost-effective wireless networking for large outdoor environments such as seaports and rail yards, where simple data transactions are required [86].

Metaradome designs should be developed and adjusted to different application areas such as maritime, telecom, radar, aerospace, and automotive applications [87]. Metaradomes may help in shaping the directivity of antennas. Directional antennas are used in wireless networks [88], and the use of metamaterials in the antenna design is described in $[88,89]$. Another application is cognitive radio. Such a radio automatically detects available channels in a frequency spectrum and accordingly changes its transmission or reception parameters to have an optimum communication bandwidth within a defined spectral region. Such a software-defined radio platform should convert into a completely reconfigurable wireless system that automatically alters its communication variables according to network and demand of user [90]. For this propose, there are studies to design suitable reconfigurable antennas [91-93].

\section{Conclusion}

The potential of metamaterials for improving the performance of microwave radomes has been outlined, and the concept of a metaradome has been presented. Several examples of 
the use of metamaterials in the design of radomes have been given. The examples discussed here are by no means the only examples possible.

The application of metamaterials to microwave radomes is a new research field which is open for further analysis and development. Potential applications are diverse and promising. Much more work is still needed in the understanding, analysis, design, and fabrication of both metamaterials and metasheets for radomes.

\section{Conflicts of Interest}

The authors declare that there are no conflicts of interest regarding the publication of this paper.

\section{References}

[1] A. B. Munk, Frequency Selective Surfaces: Theory and Design, Wiley, 2000.

[2] E. Öziş, A. V. Osipov, and T. Eibert, "Analyzing scattering and reflection from metasheets of bi-anisotropic particles," 14th Onera-DLR Aerospace Symposium, ODAS, June 2014.

[3] P. Rodriguez-Ulibarri, M. Beruete, F. Falcone et al., "Metaradome for blind spot mitigation in phased-array antennas," in Proceedings of the 8th European Conference on Antennas and Propagation, EuCAP 2014, pp. 2504-2508, Netherlands, April 2014.

[4] A. Alù, F. Bilotti, N. Engheta, and L. Vegni, "Metamaterial covers over a small aperture," IEEE Transactions on Antennas and Propagation, vol. 54, no. 6, pp. 1632-1643, 2006.

[5] M. Veysi, A. Jafargholi, and M. Kamyab, "Theory and applications of metamaterial covers," in Trends in Electromagnetism From Fundamentals to Applications, pp. 277-291, InTech, 2012.

[6] J. Hu, C.-S. Yan, and Q.-C. Lin, "A new patch antenna with metamaterial cover," Journal of Zhejiang University: Science A, vol. 7, no. 1, pp. 89-94, 2006.

[7] E. Öziş, A. V. Osipov, and T. F. Eibert, "Metamaterials for microwave radomes: an overview," Progress In Electromagnetics Research Symposium, PIERS 2015, July 2015.

[8] E. Öziş, A. V. Osipov, and T. F. Eibert, "Physical Optics and full-wave simulations of transmission of electromagnetic fields through electrically large planar meta-sheets," URSI-Landesausschuss in der Bundesrepublik Deutschland e.V., Kleinheubacher Tagung, September 2016.

[9] N. Engheta and R. W. Ziolkowski, Eds., Metamaterials: Physics and Engineering Explorations, Wiley, 2006.

[10] G. V. Eleftheriades and K. G. Balmain, Eds., Negative-Refraction Metamaterials: Fundamental Principles and Applications, Wiley, 2005.

[11] C. Caloz and T. Itoh, Electromagnetic Metamaterials: Transmission Line Theory and Microwave Applications, Wiley, 2005.

[12] L. Solymar and E. Shamonina, Waves in Metamaterials, Oxford University Press, 2009.

[13] F. Capolino, Ed., Theory and Phenomena of Metamaterials, CRC Press, 2009.

[14] R. Marqués, F. Martín, and M. Sorolla, Metamaterials with Negative Parameters: Theory, Design, and Microwave Applications, Wiley, 2008.

[15] T. J. Cui, D. R. Smith, and R. Liu, Eds., Metamaterials: Theory, Design, and Applications, Springer, 2010.
[16] W. M. Cady, M. B. Karelitz, and L. A. Turner, Radar Scanners and Radomes, Mcgraw-Hill Book Company, 1948.

[17] A. W. Rudge, K. Milne, A. D. Olver, and P. Knight, Eds., The Handbook of Antenna Design, IET, 1983.

[18] J. D. Walton, Ed., Radome Engineering Handbook, Design and Principles, Dekker, 1970.

[19] C. A. Balanis, Ed., Modern Antenna Handbook, Wiley, 2008.

[20] D. J. Kozakoff, Analysis of Radome-Enclosed Antennas, Artech House, 2010.

[21] D. F. Groutage and D. E. Smith, "Multi-layered tangent power series radome with integrated lens," in Proceedings of 14th Symposium on Electromagnetic Windows, pp. 7-11, USA, June 1978.

[22] F. R. Youngren and M. Bedford, “Tapered radomes," April 11, 1967, US patent 3,314,070.

[23] J. Pendry, "Negative $\mu$, negative $\epsilon$, negative refractive index, and how to exploit them," in Euroconference on Electromagnetic Confinement - from Basic Research to the Marketplace: Electromagnetic Crystal Structure, Scotland, June 2001.

[24] A. Sihvola, "Electromagnetic emergence in metamaterials, deconstruction of terminology of complex media," Advances in Electromagnetics of Complex Media and Metamateerials, NATO Science Series, vol. 89, pp. 3-17, 2002.

[25] T. Weiland, R. Schuhmann, R. B. Greegor et al., "Ab initio numerical simulation of left-handed metamaterials: comparison of calculations and experiments," Journal of Applied Physics, vol. 90, no. 10, pp. 5419-5424, 2001.

[26] Metamaterials home page of the future projects of the defense advanced research projects agency (DARPA), Defence Sciences Office (DSO), https://www.darpa.mil/.

[27] R. M. Walser, "Electromagnetic metamaterials," in Proceedings of the SPIE Complex Mediums II: Beyond Linear Isotropic Dielectrics, vol. 4467, pp. 1-15, 2001.

[28] S. J. Pendry, "Metamaterials and the control of electromagnetic fields," in Conference on Coherence and Quantum Optics, OSA Technical Digest, pp. 1-11, 2007.

[29] M. Durán-Sindreu, J. Naqui, F. Paredes, J. Bonache, and F. Martin, "Electrically small resonators for planar metamaterial microwave circuit and antenna design: a comparative analysis," Applied Sciences, vol. 2, no. 2, pp. 375-395, 2012.

[30] L. M. Pulido-Mancera, J. D. Baena, and J. L. Araque Quijano, "Thickness effects on the resonance of metasurfaces made of SRRs and C-SRRs," in Proceedings of IEEE Antennas and Propagation Society International Symposium, AP-S/URSI 2013, pp. 314-315, USA, July 2013.

[31] Y. Ra'di and S. A. Tretyakov, "Balanced and optimal bianisotropic particles: maximizing power extracted from electromagnetic continuous fields," New Journal of Physics, vol. 15, pp. $1-15$, June 2013.

[32] C. R. Simovski, P. A. Belov, and M. S. Kondratjev, "Electromagnetic interaction of chiral particles in three-dimensional arrays," Journal of Electromagnetic Waves and Applications, vol. 13, no. 2, pp. 189-204, 1999.

[33] J. Bonache, M. Gil, I. Gil, J. Garcia-Garcia, and F. Martin, "On the electrical characteristics of complementary metamaterial resonators," IEEE Microwave and Wireless Components Letters, vol. 16, no. 10, pp. 543-545, October 2006.

[34] A. Sihvola, "Metamaterials in electromagnetics," Metamaterials, vol. 1, pp. 2-11, February 2007.

[35] A. Priyanka, "A Review paper on metamaterial," International Journal of Engineering Sciences and Research Technology, vol. 3, no. 12, pp. 612-618, 2014. 
[36] D. M. Pozar, S. D. Targonski, and H. D. Syrigos, "Design of millimeter wave microstrip reflectarrays," IEEE Transactions on Antennas and Propagation, vol. 45, no. 2, pp. 287-296, 1997.

[37] T. K. Chang, R. J. Langley, and E. A. Parker, "Active frequencyselective surfaces," IEE Proceedings - Microwaves Antennas and Propagation, vol. 143, no. 1, pp. 62-66, March 1996.

[38] D. M. Pozar, "Flat lens antenna concept using aperture coupled microstrip patches," Electronics Letters, vol. 32, no. 23, pp. 21092111, 1996.

[39] E. Martini and S. Maci, "Metasurface Transformation Theory," in Transformation Electromagnetics and Metamaterials: Fundamental Principles and Applications, D. H. Werner and D.-H. Kwon, Eds., Springer, 2014.

[40] S. Maci, G. Minatti, M. Casaletti, and M. Bosiljevac, "Metasurfing: Addressing waves on impenetrable metasurfaces," IEEE Antennas and Wireless Propagation Letters, vol. 10, pp. 14991502, 2011.

[41] Z. Wei, Y. Cao, X. Su, Z. Gong, Y. Long, and H. Li, "Highly efficient beam steering with a transparent metasurface," Optics Express, vol. 21, no. 9, pp. 10739-10745, 2013.

[42] S. H. Mousavi, A. B. Khanikaev, J. Allen, M. Allen, and G. Shvets, "Gyromagnetically induced transparency of metasurfaces," Physical Review Letters, vol. 112, no. 11, Article ID 117402, 2014.

[43] D. Ramaccia, F. Bilotti, and A. Toscano, "Analytical model of a metasurface consisting of a regular array of sub-wavelength circular holes in a metal sheet," Progress In Electromagnetics Research M, vol. 18, pp. 209-219, 2011.

[44] S. A. Tretyakov, "Metasurfaces for general transformations of electromagnetic fields," Philosophical Transactions of the Royal Society A, vol. 373, no. 2049, Article ID 20140362, 2015.

[45] K. Achouri, B. A. Khan, S. Gupta, G. Lavigne, M. A. Salem, and C. Caloz, "Synthesis of electromagnetic metasurfaces: principles and illustrations," EPJ Applied Metamaterials, vol. 2, no. 12, pp. 1-11, 2016.

[46] V. Asadchy, M. Albooyeh, S. Tcvetkova, Y. Radi, and S. A. Tretyakov, "Metasurfaces for perfect and full control of refraction and reflection," in Proceedings of 10th International Congress on Advanced Electromagnetic Materials in Microwaves and Optics (METAMATERIALS), pp. 364-366, Greece, September 2016.

[47] T. Niemi, A. O. Karilainen, and S. A. Tretyakov, "Synthesis of polarization transformers," IEEE Transactions on Antennas and Propagation, vol. 61, no. 6, pp. 3102-3111, 2013.

[48] T. G. Mackay and A. Lakhtakia, Electromagnetic Anisotropy and Bianisotropy: A Field Guide, World Scientific Publishing Company, 2010.

[49] C. Pfeiffer and A. Grbic, "Metamaterial Huygens' surfaces: tailoring wave fronts with reflectionless sheets," Physical Review Letters, vol. 110, no. 19, Article ID 197401, 2013.

[50] H.-T. Chen, A. J. Taylor, and N. Yu, "A review of metasurfaces: physics and applications," Reports on Progress in Physics, pp. 144, 2016.

[51] A. Alù, "Viewpoint: wave-shaping surfaces," Physics, 2013, https://physics.aps.org/articles/v6/53.

[52] J. B. Pendry and D. R. Smith, "The quest for the superlens," Scientific American, vol. 295, no. 1, pp. 60-67, 2006.

[53] A. E. Culhaoglu, A. V. Osipov, and P. Russer, "Imaging by a double negative metamaterial slab excited with an arbitrarily oriented dipole," Radio Science, vol. 49, no. 1, pp. 68-79, 2014.
[54] J. P. Turpin, J. A. Bossard, K. L. Morgan, D. H. Werner, and P. L. Werner, "Reconfigurable and tunable metamaterials: a review of the theory and applications," International Journal of Antennas and Propagation, Article ID 429837, pp. 1-18, 2014.

[55] A. Ahmed, I. A. Goldthorpe, and A. K. Khandani, "Electrically tunable materials for microwave applications," Applied Physics Reviews, vol. 2, no. 1, Article ID 011302, 2015.

[56] Z. Sheng and V. V. Varadan, "Tuning the effective properties of metamaterials by changing the substrate properties," Journal of Applied Physics, vol. 101, no. 1, Article ID 014909, 2007.

[57] M. Barbuto, A. Monti, F. Bilotti, and A. Toscano, "Design of a non-foster actively loaded SRR and application in metamaterial-inspired components," IEEE Transactions on Antennas and Propagation, vol. 61, no. 3, pp. 1219-1227, 2013.

[58] D. Bouyge, D. Mardivirin, J. Bonache et al., "Split ring resonators (SRRs) based on micro-electro-mechanical deflectable cantilever-type rings: Application to tunable stopband filters," IEEE Microwave and Wireless Components Letters, vol. 21, no. 5, pp. 243-245, 2011.

[59] T. Debogovic and J. Perruisseau-Carrier, "MEMS-reconfigurable metamaterials and antenna applications," International Journal of Antennas and Propagation, vol. 2014, Article ID 138138, 8 pages, 2014.

[60] D. Peroulis, K. Sarabandi, and L. P. B. Katehi, "Design of reconfigurable slot antennas," IEEE Transactions on Antennas and Propagation, vol. 53, no. 2, pp. 645-654, 2005.

[61] S. Shelley, J. Costantine, C. G. Christodoulou, D. E. Anagnostou, and J. C. Lyke, "FPGA-controlled switch-reconfigured antenna," IEEE Antennas and Wireless Propagation Letters, vol. 9, pp. 355$358,2010$.

[62] D. Huang, E. Poutrina, and D. R. Smith, "Analysis of the power dependent tuning of a varactor-loaded metamaterial at microwave frequencies," Applied Physics Letters, vol. 96, no. 10, Article ID 104104, 2010.

[63] E. Özbay, K. Aydin, S. Butun, K. Kolodziejak, and D. Pawlak, "Ferroelectric based tuneable SRR based metamaterial for microwave applications," in Proceedings of European Microwave Conference, EUMC, pp. 497-499, Germany, October 2007.

[64] D. Shrekenhamer, W.-C. Chen, and W. J. Padilla, "Liquid crystal tunable metamaterial absorber," Physical Review Letters, vol. 110, no. 17, Article ID 177403, 2013.

[65] Q. Zhao, L. Kang, B. Du et al., "Electrically tunable negative permeability metamaterials based on nematic liquid crystals," Applied Physics Letters, vol. 90, no. 1, Article ID 011112, 2007.

[66] A. Ebrahimi, P. Yaghmaee, W. Withayachumnankul, C. Fumeaux, S. Al-Sarawi, and D. Abbott, "Interlayer tuning of $\mathrm{X}$-band frequency-selective surface using liquid crystal," in Proceedings of 3rd Asia-Pacific Microwave Conference, APMC 2013, pp. 1118-1120, Korea, November 2013.

[67] C. Kitte, "Introduction to Solid State Physics," in Wiley Series on the Science and Technology of Materials, Second edition, 1961.

[68] K. Bi, G. Dong, X. Fu, and J. Zhou, "Ferrite based metamaterials with thermo-tunable negative refractive index," Applied Physics Letters, vol. 103, no. 13, Article ID 131915, 2013.

[69] G. Oliveri, D. Werner, F. Bilotti, and C. Craeye, "Reconfigurable electromagnetics through metamaterials," International Journal of Antennas and Propagation, vol. 2014, Article ID 215394, 2 pages, 2014.

[70] T.-C. Pu, H.-H. Lin, C.-Y. Wu, and J.-H. Chen, "Photovoltaic panel as metamaterial antenna radome for dual-band application," Microwave and Optical Technology Letters, vol. 53, no. 10, pp. 2382-2388, 2011. 
[71] H. Boutayeb and T. A. Denidni, "Internally excited fabry-Pérot type cavity: power normalization and directivity evaluation," IEEE Antennas and Wireless Propagation Letters, vol. 5, no. 1, pp. 159-162, 2006.

[72] R. Basiry, H. Abiri, and A. Yahaghi, "Electromagnetic performance analysis of omega-type metamaterial radomes," International Journal of RF and Microwave Computer-Aided Engineering, vol. 21, no. 6, pp. 665-673, 2011.

[73] H.-N. Liu, H.-L. Su, K.-H. Lin, C.-Y. Wu, C.-L. Tang, and S.H. Yeh, "Design of antenna radome composed of metamaterials for high gain," in Proceedings of IEEE Antennas and Propagation Society International Symposium, APS 2006, pp. 19-22, USA, July 2006.

[74] H. Cory, Y. J. Lee, Y. Hao, and C. G. Parini, "Use of conjugate dielectric and metamaterial slabs as radomes," IET Microwaves, Antennas and Propagation, vol. 1, no. 1, pp. 137-143, 2007.

[75] K.-S. Chen, K.-H. Lin, and H.-L. Su, "Microstrip antenna gain enhancement by metamaterial radome with more subwavelength holes," in Proceedings of Asia Pacific Microwave Conference 2009, APMC 2009, pp. 790-792, Singapore, December 2009.

[76] T. Crépin, C. Martel, B. Gabard et al., "Blind spot mitigation in phased array antenna using metamaterials," in Proceedings of International Radar Conference, Radar 2014, 4 pages, France, October 2014.

[77] D. M. Pozar and D. H. Schaubert, "Scan blindness in infinite phased arrays of printed dipoles," IEEE Transactions on Antennas and Propagation, vol. 32, no. 6, pp. 602-610, 1984.

[78] M. Latrach, H. Rmili, C. Sabatier, E. Seguenot, and S. Toutain, "Design of a new type of metamaterial radome for low frequencies," Microwave and Optical Technology Letters, vol. 52, no. 5, pp. 1119-1123, 2010.

[79] H.-L. Su, H.-C. Huang, K.-H. Lin, C.-Y. Wu, and H.-H. Lin, "Gain-enhanced metamaterial radome for circularly-polarized antenna," in Proceedings of IEEE International Symposium on Antennas and Propagation and CNC-USNC/URSI Radio Science Meeting, AP-S/URSI 2010, pp. 661-664, Canada, July 2010.

[80] X. G. Zhang, X. G. Miao, X. W. Fang et al., "Artificial ceramic metamaterial with meshed grid structure for radome application," Advanced Materials Research, vol. 893, pp. 11-14, 2014.

[81] R. Dubrovka, G. Palikaras, and P. Belov, "Near-field antenna radome based on extremely anisotropic metamaterial," IEEE Antennas and Wireless Propagation Letters, vol. 11, pp. 438-441, 2012.

[82] A. V. Osipov, E. Kemptner, E. Özis, and H. Kobayashi, "Microwave metamaterials for increased survivability of future systems," IEICE Technical Report, vol. 116, no. 427, pp. 35-40, January 2017.

[83] T. Jiang, Z. Wang, D. Li et al., "Low-DC voltage-controlled steering-antenna radome utilizing tunable active metamaterial," IEEE Transactions on Microwave Theory and Techniques, vol. 60, no. 1, pp. 170-178, 2012.

[84] V. G. Harris, A. Geiler, Y. Chen et al., "Recent advances in processing and applications of microwave ferrites," Journal of Magnetism and Magnetic Materials, vol. 321, no. 14, pp. 2035-2047, 2009.

[85] A. Parsa, T. Kodera, and C. Caloz, "Ferrite based non-reciprocal radome, generalized scattering matrix analysis and experimental demonstration," IEEE Transactions on Antennas and Propagation, vol. 59, no. 3, pp. 810-817, 2011.

[86] White Paper, Narrowband technology: the most intelligent choice for wireless connectivity in the most expansive outdoor spaces
(Choosing the right wireless network for expansive outdoor spaces), Zebra Technologies, 2015.

[87] D. J. Kozakoff, "Multi-frquency and ultrawideband antenna radomes," Antenna Systems 2011, USA, September 2011, available at http://www.slideserve.com/duy/multi-frequencyand-ultrawideband-antenna-radomes.

[88] R.-X. Wu, Y. Gu, and L. Tan, "Optimizing the directional radiation of metamaterial antenna," in Proceedings of 7th International Congress on Advanced Electromagnetic Materials in Microwaves and Optics, METAMATERIALS 2013, pp. 106-108, France, September 2013.

[89] L.-M. Si and X. Lv, "CPW-fed multi-band omni-directional planar microstrip antenna using composite metamaterial resonators for wireless communications," Progress in Electromagnetics Research, vol. 83, pp. 133-146, 2008.

[90] P. P. Bhattacharya, R. Khandelwal, R. Gera, and A. Agarwal, "Smart radio spectrum management for cognitive radio," International Journal of Distributed and Parallel Systems (IJDPS), vol. 2, no. 4, pp. 12-24, July 2011.

[91] P.-Y. Qin, F. Wei, and Y. J. Guo, "A Wideband-to-Narrowband Tunable Antenna Using A Reconfigurable Filter," IEEE Transactions on Antennas and Propagation, vol. 63, no. 5, pp. 2282-2285, 2015.

[92] Y. Tawk and C. G. Christodoulou, "A new reconfigurable antenna design for cognitive radio," IEEE Antennas and Wireless Propagation Letters, vol. 8, pp. 1378-1381, 2009.

[93] G. Prema and P. Gayatri, "A frequency reconfigurable rotatable antenna design for cognitive radio systems," International Journal of Engineering Research and Technology (IJERT), vol. 2, no. 12, pp. 1342-1349, December 2013.

[94] Y. Liu and X. Zhang, "Metamaterials: a new frontier of science and technology," Chemical Society Reviews, vol. 40, no. 5, pp. 2494-2507, 2011.

[95] O. Vanbesien, Artificial Materials, Wiley, 2012.

[96] C. R. Simovski, M. S. Kondratiev, P. A. Belov, and S. A. Tretyakov, "Excitation dyadics for the grids of chiral and omega particles," in Proceedings of SPIE the Smart Structures and Materials 1997: Mathematics and Control in Smart Structures, vol. 3039, pp. 692-703, USA, March 1997.

[97] J. A. Gordon, C. L. Holloway, and A. Dienstfrey, "A physical explanation of angle-independent reflection and transmission properties of metafilms/metasurfaces," IEEE Antennas and Wireless Propagation Letters, vol. 8, pp. 1127-1130, 2009. 


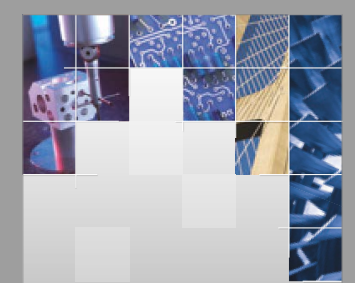

\section{Enfincering}
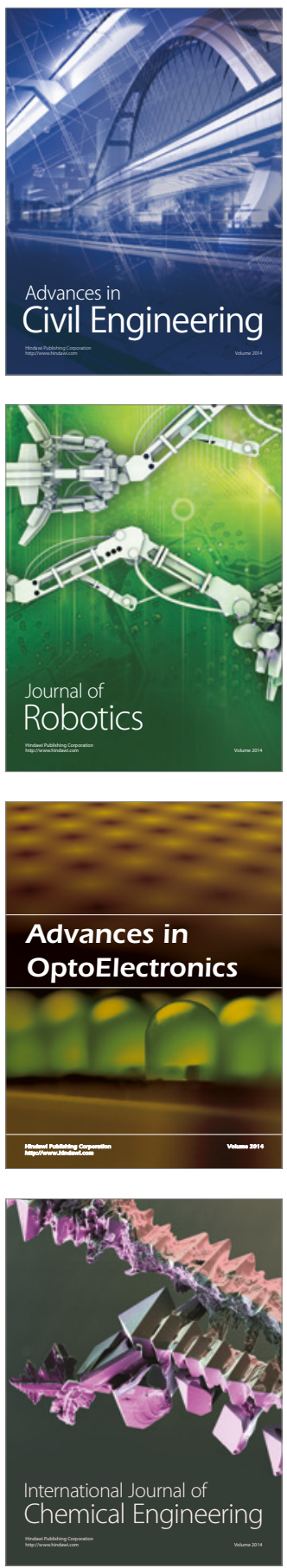

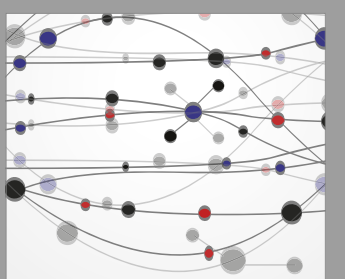

The Scientific World Journal

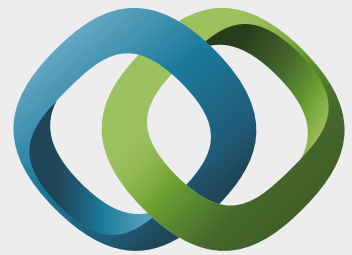

\section{Hindawi}

Submit your manuscripts at

https://www.hindawi.com
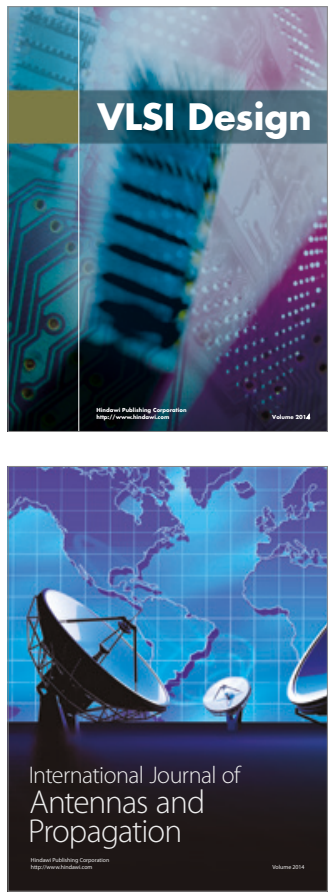

\section{Rotating}

Machinery
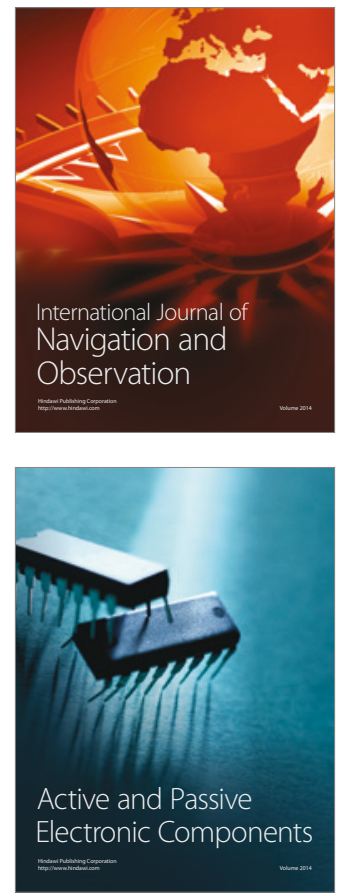
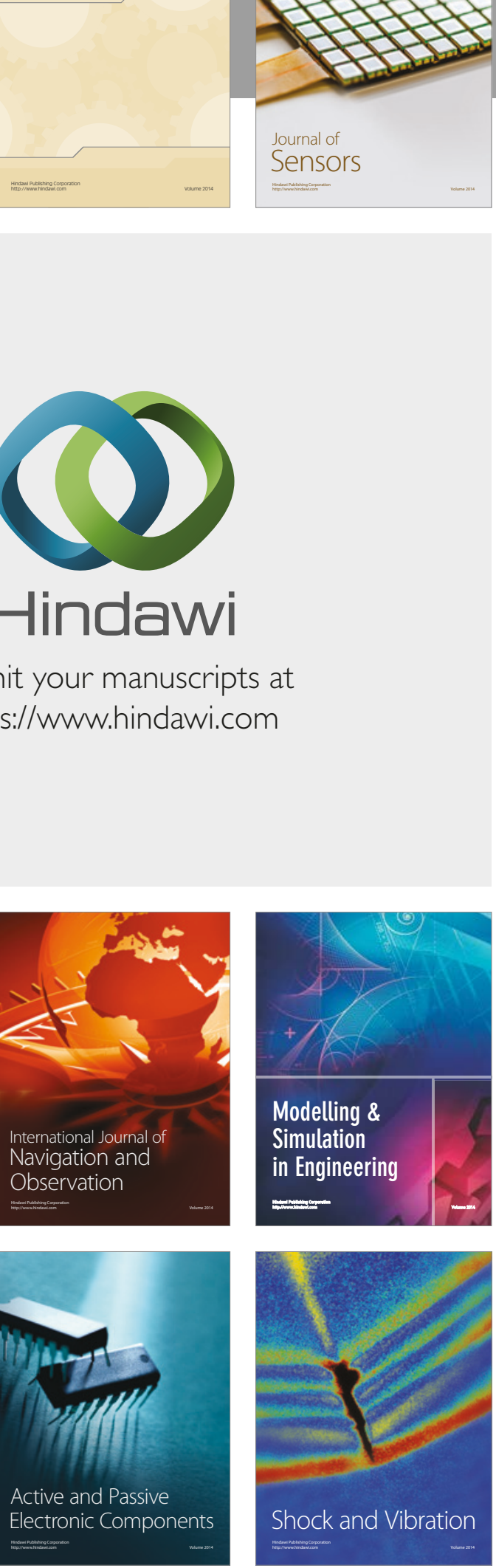
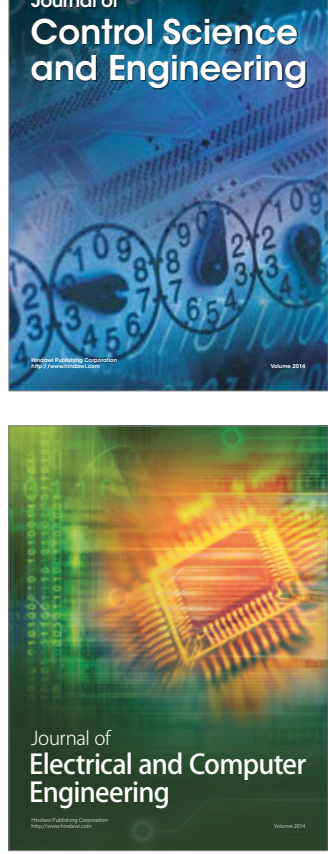

Distributed

Journal of

Control Science

and Engineering
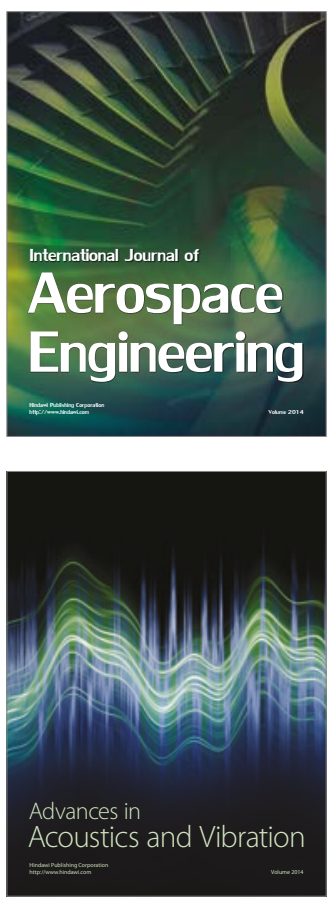

Sensor Networks 